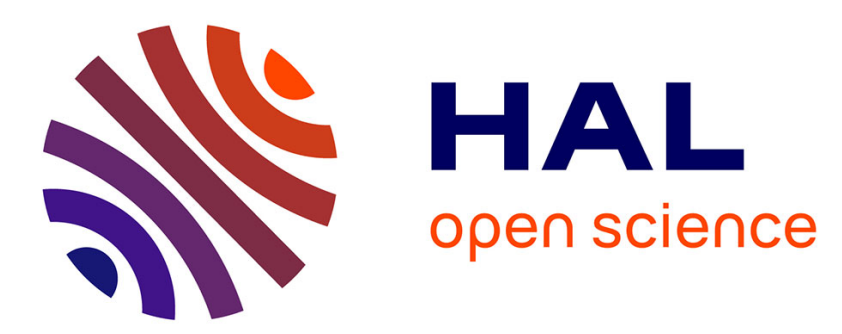

\title{
Contrôle du dopage dans la croissance épitaxiale d'arséniure de gallium
}

\author{
A. Boucher, J.P. Chané, E. Fabre
}

\section{To cite this version:}

A. Boucher, J.P. Chané, E. Fabre. Contrôle du dopage dans la croissance épitaxiale d'arséniure de gallium. Revue de Physique Appliquée, 1971, 6 (1), pp.5-10. 10.1051/rphysap:01971006010500 . jpa-00243504

\section{HAL Id: jpa-00243504 https://hal.science/jpa-00243504}

Submitted on 1 Jan 1971

HAL is a multi-disciplinary open access archive for the deposit and dissemination of scientific research documents, whether they are published or not. The documents may come from teaching and research institutions in France or abroad, or from public or private research centers.
L'archive ouverte pluridisciplinaire HAL, est destinée au dépôt et à la diffusion de documents scientifiques de niveau recherche, publiés ou non, émanant des établissements d'enseignement et de recherche français ou étrangers, des laboratoires publics ou privés. 


\title{
CONTRÔLE DU DOPAGE DANS LA CROISSANCE ÉPITAXIALE D'ARSÉNIURE DE GALLIUM
}

\author{
A. BOUCHER, J. P. CHANÉ et E. FABRE \\ Laboratoire d'Electronique et de Physique Appliquée \\ 94, Limeil - Brévannes
}

(Reçu le 17 août 1970)

\begin{abstract}
Résumé. - Nous présentons nos résultats récemment acquis dans la maîtrise du niveau et du profil de dopage de couches d'arséniure de gallium préparées par épitaxie en phase vapeur. Nous décrivons un procédé de dopage par rétrodiffusion, et exposons nos méthodes de caractérisation.

Abstract. - In this paper we shall give our last results on the setting of the doping level and the concentration profile of vapor grown Gallium Arsenide epitaxial layers.

An operating process of doping by back-diffusion and our methods of characterization are given.
\end{abstract}

Les exigences actuelles de puissance et de fiabilité de dispositifs hyperfréquences réalisés à partir de couches épitaxiales d'arséniure de Gallium, imposent de la part du matériau des caractéristiques améliorées concernant en particulier sa mobilité, son niveau et son profil de dopage.

L'optimalisation de ces caractéristiques sous-entend un effort important, visant à maîtriser les conditions de croissance et de dopage ; on parvient alors à un niveau constant dans la qualité du matériau.

Nous donnerons dans cet article nos résultats récemment acquis dans ce domaine.

1. Procédés de dopage. - La méthode de croissance épitaxiale que nous avons utilisée est basée sur le transport du gallium en phase vapeur par le trichlorure d'arsenic [1] [2]. L'équilibre qui s'établit alors entre les différents constituants gazeux au niveau de la source de gallium (portée à une température de $850^{\circ} \mathrm{C}$ ) se trouve déplacé dans le sens favorable à la formation d'arséniure de gallium, lorsque les gaz entraînés par l'hydrogène vecteur arrivent au voisinage des substrats placés à $750^{\circ} \mathrm{C}$ [3].

Le dopage de la couche déposée a lieu lorsqu'une faible pression partielle du dopant est assurée dans le flux gazeux responsable du transport.

Jusqu'à présent deux moyens permettaient d'obtenir ce résultat.

Le premier, utilisé pour le dopage au soufre, consiste à mettre en parallèle avec le circuit de croissance, un second circuit amenant des vapeurs dopantes en très faible concentration.

Cette opération est délicate car il est difficile d'assurer une pression partielle à la fois faible et bien contrôlée.

Le second moyen consiste à introduire dans la source de Gallium un dopant métallique (par exemple une quantité connue d'étain).

Enfin, nous venons de mettre au point un troisième procédé, utilisant le phénomène de la rétrodiffusion gazeuse [4].

PRINCIPE DE DOPAGE PAR RÉTRODIFFUSION. - Si en des points $\mathrm{A}$ et $\mathrm{B}$ d'une enceinte (Fig. 1) arrivent

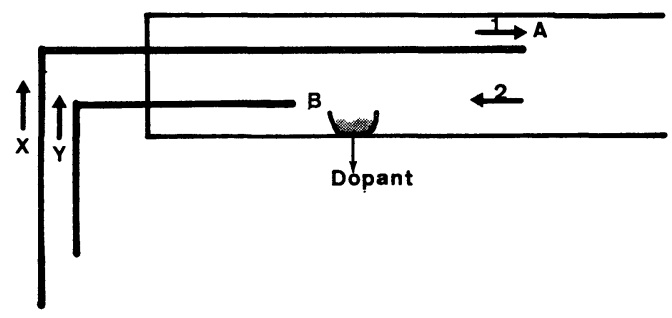

FIG. 1. - Schéma de principe du dopage par rétrodiffusion gazeuse.

simultanément des gaz $\mathrm{X}$ et $\mathrm{Y}$ dans le sens 1 , il se produit une rétrodiffusion dans le sens 2 d'une fraction du gaz $X$ vers le point $B$. Cette rétrodiffusion est fonction du rapport des débits des gaz $X$ et $Y$.

En plaçant alors entre $A$ et $B$ un élément dopant susceptible de réagir avec le gaz rétrodiffusant (sans réagir avec le gaz vecteur $Y$ ) on constate l'attaque du dopant et sa présence dans le flux gazeux s'écoulant selon le sens 1. Le dopage qui en résulte est alors fonction de la rétrodiffusion, elle-même fonction exponentielle de la position du dopant dans le réacteur ; ce qui permet un bon contrôle, notamment pour les très grandes dilutions. Pour obtenir une valeur déterminée du niveau de dopage, il suffit alors de régler le débit du gaz vecteur $Y$, tout en maintenant constant de débit du gaz $X$.

Cependant, l'application de ce phénomène au contrôle du dopage exige que la tension de vapeur du dopant soit négligeable à la température de l'enceinte (afin que le transport du dopant résulte uniquement de l'attaque chimique par le gaz $X$ rétrodiffusé). 
En respectant cette condition et en fixant parfaitement les divers paramètres (voir le chapitre suivant), il est possible de tracer une courbe d'étalonnage du niveau de dopage en fonction du débit de gaz vecteur $Y$ (Fig. 2).

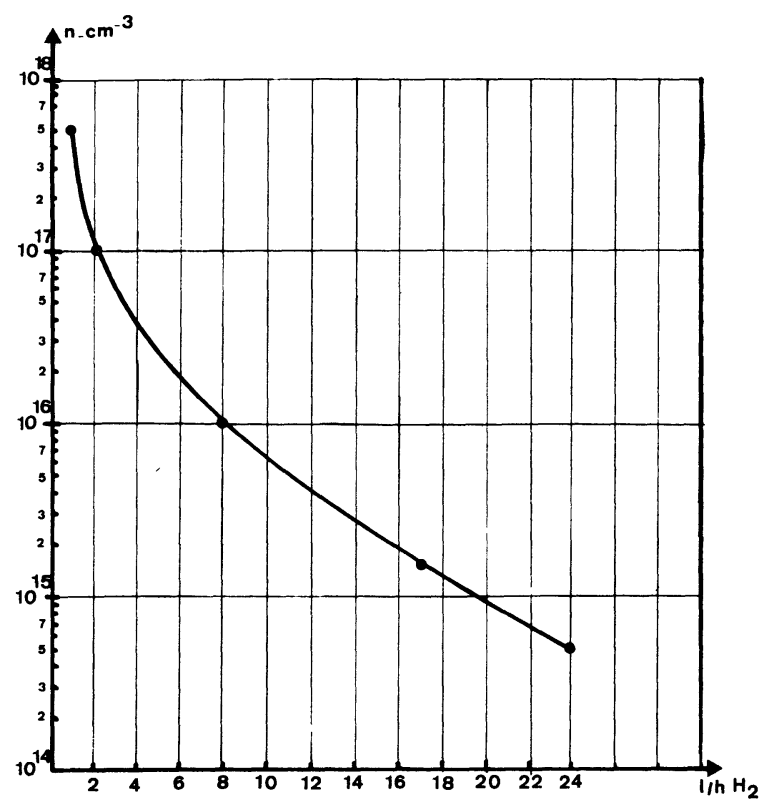

Fig. 2. - Exemple de courbe d'étalonnage du système de dopage.

Cette courbe n'est bien entendu valable que si le débit de gaz $\mathbf{X}$ est constant et l'abscisse du creuset contenant le dopant, fixe. Cette abscisse doit être déterminée expérimentalement pour optimaliser toutes les conditions (réglage facile et effet de mémoire minimum).

II. Niveau de dopage. - En utilisant l'un ou l'autre des procédés précédemment cités ou décrits, nous pouvons fixer de façon reproductible le dopage de type $n$ dans nos couches épitaxiales, pour $n$ variant entre $10^{15}$ et $5 \times 10^{18}$ atomes. $\mathrm{cm}^{-3}$. A titre d'exemple, nous avons consigné dans le tableau I la concentration en porteurs libres (déterminée par mesure d'effet Hall à $300^{\circ} \mathrm{K}$ et à $77^{\circ} \mathrm{K}$ ) d'une série de 17 épitaxies consécutives suivant le procédé de dopage par rétrodiffusion, en maintenant aussi constants que possible les paramètres suivants :

- substrats (qualité-orientation-préparation),

- pureté des matières premières,

- mode opératoire concernant le décapage et la croissance.

Les données statistiques de cette série sont les suivantes :

- dopage moyen : $2,07 \times 10^{15}$ atomes. $\mathrm{cm}^{-3}$,

- écart-type estimé : $0,48 \times 10^{15}$ atomes. $\mathrm{cm}^{-3}$.

On remarque tout d'abord que le niveau de dopage est proche de l'optimum recherché pour les diodes GUNN oscillant en bande X.

La valeur de l'écart-type témoigne d'une maîtrise
TABLEAU I

Suite d'expériences consécutives. Reproductibilité de $n$ et $\mu$ à 293 et $77 \circ \mathrm{K}$. Mesures obtenues en caractérisation par effet Hall. Echantillon 309* caractérisé par diode Schottky

\begin{tabular}{|c|c|c|c|c|c|}
\hline $\mathrm{N}^{\circ}$ essai & $n 293{ }^{\circ} \mathrm{K}$ & $\mu 293^{\circ} \mathrm{K}$ & $n$ & $\underline{77}^{\circ} \mathrm{K}$ & $\stackrel{\mu 7^{\circ} \mathrm{K}}{-}$ \\
\hline Ep. II 303 & $1,3 \times 10^{15}$ & 7700 & 1 & $\times 10^{15}$ & 458 \\
\hline Ep. II 304 & $2,3 \times 10^{15}$ & 7000 & 1,8 & $\times 10^{15}$ & 35600 \\
\hline Ep. II 305 & $\times 10^{15}$ & 7900 & 1,64 & $4 \times 10^{15}$ & 45300 \\
\hline Ep. II 306 & $1,15 \times 10^{15}$ & 7600 & 9,4 & $\times 10^{14}$ & 52500 \\
\hline Ep. II 307 & $2,3 \times 10^{15}$ & 7330 & 1,8 & $\times 10^{15}$ & 40500 \\
\hline Ep. II 308 & $1,55 \times 10^{15}$ & 7350 & 1,06 & $5 \times 10^{15}$ & 38600 \\
\hline Ep. II 309* & $\times 10^{15}$ & - & & - & - \\
\hline Ep. II 310 & $2,16 \times 10^{15}$ & 7420 & 1,67 & $7 \times 10^{15}$ & 37800 \\
\hline Ep. II 311 & $\times 10^{15}$ & 7300 & 1,5 & $\times 10^{15}$ & 46400 \\
\hline Ep. II 313 & $1,65 \times 10^{15}$ & 7600 & 1,31 & $1 \times 10^{15}$ & 45000 \\
\hline Ep. II 314 & $2,14 \times 10^{15}$ & 7900 & 1,72 & $2 \times 10^{15}$ & 47300 \\
\hline Ep. II 316 & $1,97 \times 10^{15}$ & 7800 & 1,61 & $1 \times 10^{15}$ & 49000 \\
\hline Ep. II 317 & $1,78 \times 10^{15}$ & 7800 & 1,44 & $4 \times 10^{15}$ & 51500 \\
\hline Ep. II 318 & $3,0 \times 10^{15}$ & 7400 & 2,3 & $\times 10^{15}$ & 43500 \\
\hline Ep. II 319 & $1,7 \times 10^{15}$ & 7700 & 1,35 & $5 \times 10^{15}$ & 47000 \\
\hline Ep. II 321 & $\times 10^{15}$ & 8200 & 1,7 & $\times 10^{15}$ & 49000 \\
\hline p. II 322 & $2,33 \times 10^{15}$ & 7780 & 1,88 & $8 \times 10^{15}$ & 44300 \\
\hline p. II 323 & $2,8 \times 10^{15}$ & 7600 & 2,24 & $4 \times 10^{15}$ & 41600 \\
\hline
\end{tabular}

$\begin{array}{ll}\eta=2,7 & \text { pour l'Ep. II } 307 \\ \eta=3,2 & \text { pour l'Ep. II } 321\end{array}$

satisfaisante $\mathrm{du}$ dopage qui fut progressivement acquise, non seulement par l'emploi du procédé de dopage par rétrodiffusion, mais aussi en contrôlant les conditions de début de croissance (concernant le réacteur et les substrats) et les conditions du décapage " in situ " (ce décapage dans le réacteur, précède immédiatement le dépôt ; il est provoqué par le gaz chlorhydrique issu de la décomposition du trichlorure d'Arsenic dans un circuit annexe).

III. Profil de dopage. - Précisons d'abord que la caractérisation des couches épitaxiales peut se faire soit sur substrat semi-isolant (méthode de Hall avec décapages successifs) soit sur substrat $n^{+}$(mesures capacitives sur diodes Schottky). La description de ces méthodes sera faite au paragraphe suivant. Des mesures effectuées par chacune d'elles sur des couches obtenues au cours de la même opération ont donné des profils pratiquement identiques (Fig. 3).

Volontairement, nous ne tiendrons pas compte des premiers microns de croissance. En effet, les problèmes liés à la fois aux phénomènes existant au niveau de l'interface, et à l'influence du substrat sont très complexes, et font l'objet d'une autre publication [5].

Cette restriction faite, on constate grâce à une caractérisation systématique, que la couche $n$ possède un profil de dopage très reproductible, pouvant d'ailleurs être modifié suivant le type d'application auquel le matériau épitaxié est destiné.

Pour parvenir à un tel contrôle, il a fallu procéder à de nombreux essais préliminaires afin de définir les conditions précises de croissance et de dopage. 


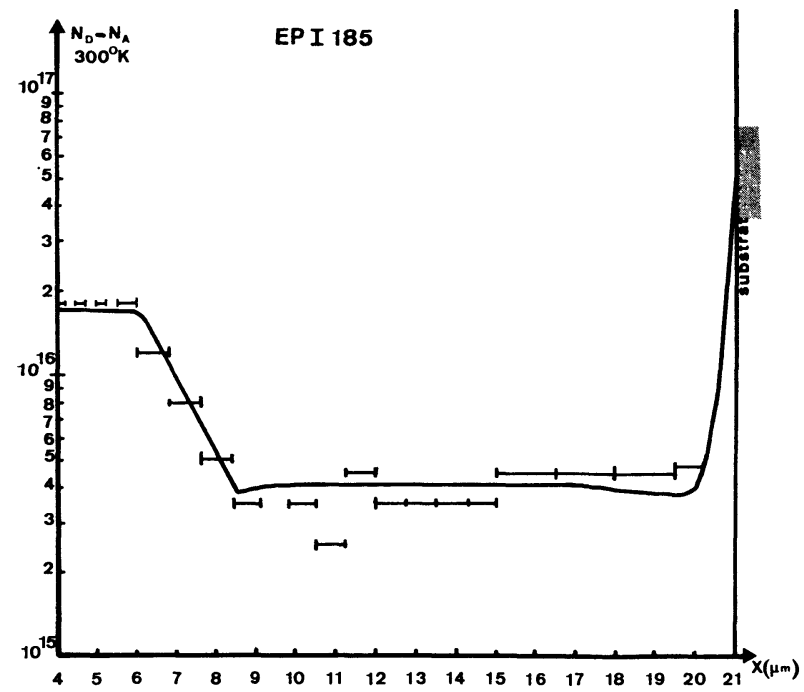

Fig. 3. - Comparaison des profils obtenus par mesures d'effet Hall avec décapages successifs sur substrat semi-isolant (-), et par diodes Schottky sur substrat $n^{+}$(courbe en traits pleins).

Si nous recherchons un profil plat, l'écart ne dépasse pas $10 \%$ sur une profondeur de $20 \mu \mathrm{m}$. A titre d'exemple nous donnons (Fig. 4) le profil de trois couches épitaxiales différentes qui répondent chacune à des applications précises.

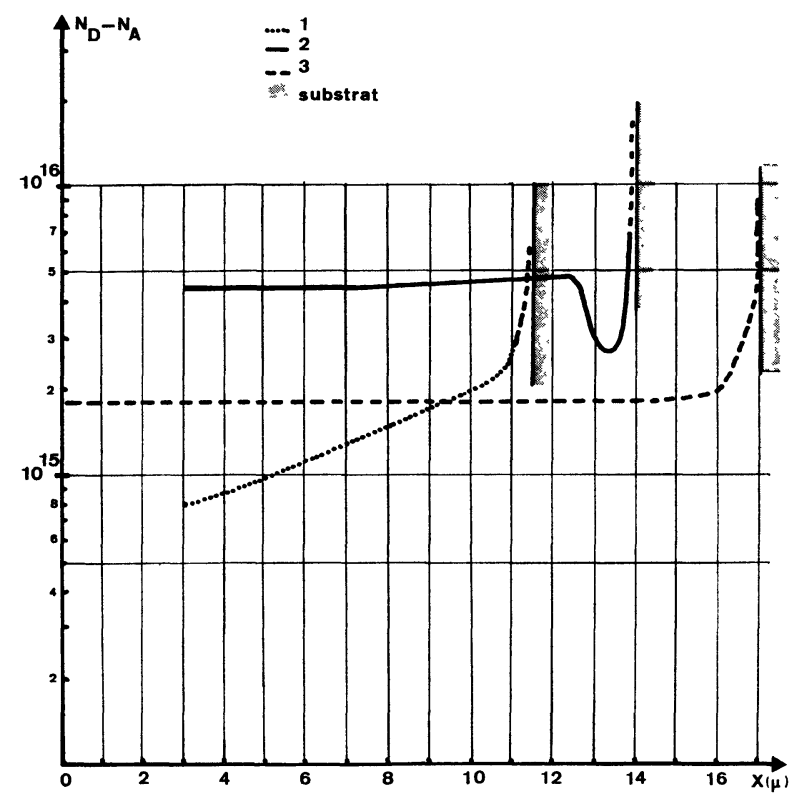

Fig. 4. - Différents types de profil : Le profil de type 2 est fréquemment rencontré sur substrat $n^{+}$dopé silicium, alors que les profils 1 et 3 sont généralement obtenus sur substrat $n^{+}$ dopé tellure.

StRUCTURES MUlticouches. - Cette maîtrise du profil de dopage s'avère très utile dans l'élaboration de structures multicouches $\left(n^{+} / n /\right.$ substrat $)$, que cellesci soient faites en une ou deux opérations successives. Dans ce dernier cas, il s'agit de déposer une couche $n^{+}$sur une épitaxie $n$ obtenue dans un autre réacteur. Si les conditions sont en principe les mêmes, le décapage «in situ » doit être parfaitement maîtrisé afin
Structure sandwich ${ }_{51}^{n++} / n^{+} / n / n^{+}$

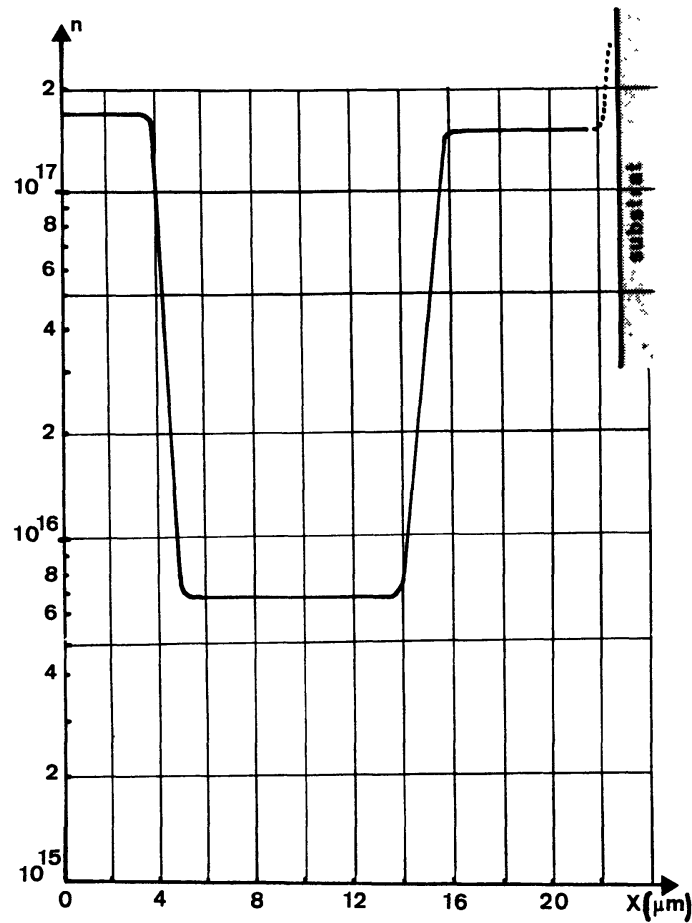

FIG. 5. - Structures multicouches.

de garder une épaisseur déterminée à la couche $n$ sous-jacente. Actuellement, l'incertitude sur nos décapages est de l'ordre du micron. Quant aux caractéristiques de la couche $n^{+}$, elles se situent d'une façon reproductible au voisinage de $2 \times 10^{18}$ atomes. $\mathrm{cm}^{3}$, avec une mobilité de $2300 \mathrm{~cm}^{2} \cdot \mathrm{V}^{-1} \cdot \mathrm{s}^{-1}$.

La méthode de dopage par rétrodiffusion permet l'élaboration des multicouches en une seule opération. Il suffit de faire varier brutalement les conditions de dopage pour modifier le profil entre les valeurs extrêmes de $10^{15}$ à $5 \times 10^{17}$ atomes. $\mathrm{cm}^{-3}$ et ceci avec une grande souplesse d'utilisation et pratiquement aucune inertie. Les jonctions obtenues sont abruptes comme le montre la structure $n^{+} / n / n^{+}$substrat de la figure $5[3]$.

IV. Caractérisation des couches épitaxiales. - 1) DÉTERMINATION DES PROFILS. - Dans toutes les opérations d'épitaxie, la même couche est déposée sur deux substrats de type différent, l'un semi-isolant et l'autre $n^{+}$.

a) Sur substrat semi-isolant. - On détermine par effet Hall les propriétés électriques du matériau résistivité, mobilité, concentration des porteurs. Ces mesures sont faites systématiquement pour toutes les épitaxies, à $300^{\circ} \mathrm{K}$ et à $77^{\circ} \mathrm{K}$.

On peut aussi déterminer le profil de concentration $n(x)$ du matériau en effectuant des décapages successifs de la couche, entre deux mesures d'effet Hall. Affectons l'indice $i$ aux différents paramètres avant le 
ième décapage, et l'indice $(i+1)$ aux mêmes paramètres après le ième décapage.

Soient donc $V_{i}$ et $V_{i+1}$ les tensions de Hall mesurées, $n_{i}$ et $n_{i+1}$ les concentrations en porteurs libres dans les couches d'épaisseur $d_{i}$ et $d_{i+1}$ (Fig. 6).

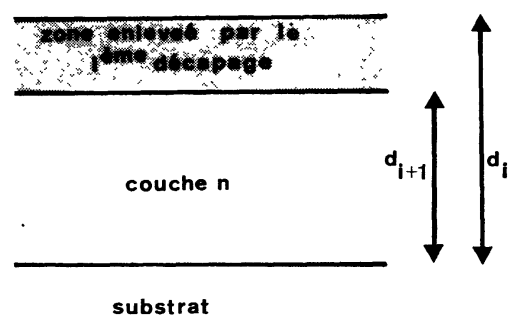

S.I.

FIG. 6. - Décapages successifs pour la détermination de profil par effet Hall:

On a toujours :

$$
R_{\mathrm{H}_{i}}=\frac{1}{n_{i} e}=\frac{V_{i} d_{i}}{I . B} \cdot 10^{8},
$$

$I$ étant l'intensité qui parcourt l'échantillon, $B$ le champ magnétique et $e$ la charge électronique, on en déduit :

$$
\begin{aligned}
n_{i} & =\frac{1}{e} \frac{I \cdot B \cdot 10^{-8}}{V_{i} \cdot d_{i}} \\
n_{i+1} & =\frac{1}{e} \frac{I \cdot B \cdot 10^{-8}}{V_{i+1} \cdot d_{i+1}} .
\end{aligned}
$$

Soit $N$ le nombre total de porteurs, dans tout l'échantillon :

$N i=n_{i} \cdot S \cdot d_{i}, \quad S$ étant la surface de l'échantillon $N_{i+1}=n_{i+1} \cdot S \cdot d_{i+1}$.

On en déduit le nombre de porteurs $n$ par unité de volume, dans la partie décapée :

$$
\begin{aligned}
& n=\frac{N_{i}-N_{i+1}}{S \cdot\left(d_{i}-d_{i+1}\right)}=\frac{n_{i} \cdot d_{i}-n_{i+1} \cdot d_{i+1}}{d_{i}-d_{i+1}} \\
& n=\frac{I \cdot B \cdot 10^{-8}}{e}\left(\frac{1}{V_{i}}-\frac{1}{V_{i+1}}\right) \frac{1}{d_{i}-d_{i+1}}
\end{aligned}
$$

Si on effectue des décapages successifs d'épaisseur égale, $\left(d_{i}-d_{i+1}\right)$ est une constante, et

$$
n=A\left(\frac{1}{V_{i}}-\frac{1}{V_{i+1}}\right)
$$

$A$ étant une constante.

Une mesure précise des tensions de Hall avant et après chaque décapage permet de calculer la concentration dans la partie décapée, et donc de déterminer le profil de concentration à l'intérieur de la couche, depuis la surface jusqu'au substrat (Fig. 3).

b) Sur substrat $n^{+}$. - On détermine le profil de concentration par mesure de la variation de la capacité d'une diode Schottky déposée sur le matériau, en fonction de la polarisation inverse appliquée à cette diode. Mais la profondeur de couche épitaxiale que permet d'explorer une diode étant très faible (quelques microns si le dopage est voisin de $10^{15}$ atomes. $\mathrm{cm}^{-3}$ ), il est nécessaire d'effectuer des séries de mesures sur plusieurs diodes déposées à des profondeurs différentes dans la couche, si l'on veut obtenir le profil complet de concentration. Nous avons résolu le problème en mettant au point une méthode de décapage «en biseau ॥ de la couche épitaxiale [6].

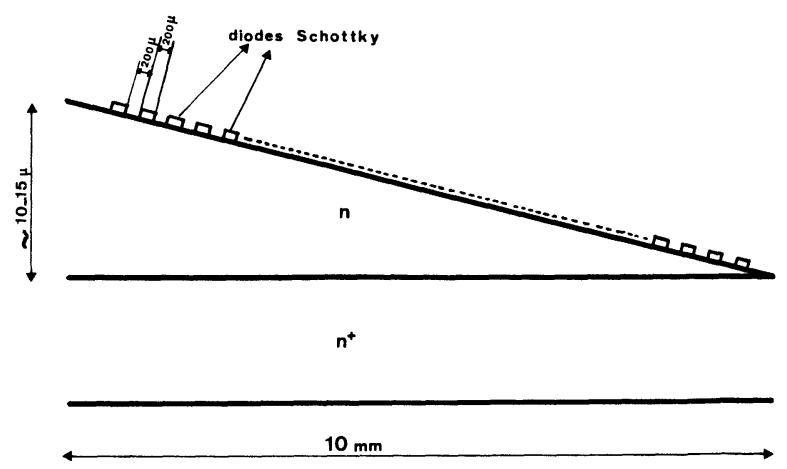

FIG. 7. - Détermination des profils par diodes Schottky déposées sur un biseau.

On dépose alors sur la couche décapée un peigne de diodes Schottky de $1 \mathrm{~mm}$ de long sur $200 \mu$ de large, au pas de $200 \mu$, depuis la surface jusqu'à l'interface (Fig. 7). On prend un contact successivement sur chaque diode à l'aide d'un micromanipulateur, et un second contact, allié, sur le substrat $n^{+}$. Le profil de concentration obtenu sous chaque diode se recouvre parfaitement avec celui obtenu avec les diodes qui lui sont voisines. C'est par cette méthode qu'ont été tracés les profils des figures 4 et 5 .

2) Mobilité Des COUCHes obTenues. CoMPENSATION. - Sur la figure 8, nous avons porté la mobilité des couches épitaxiales en fonction de leur concentration, ces deux paramètres étant déterminés par mesure d'effet Hall à la température ambiante.

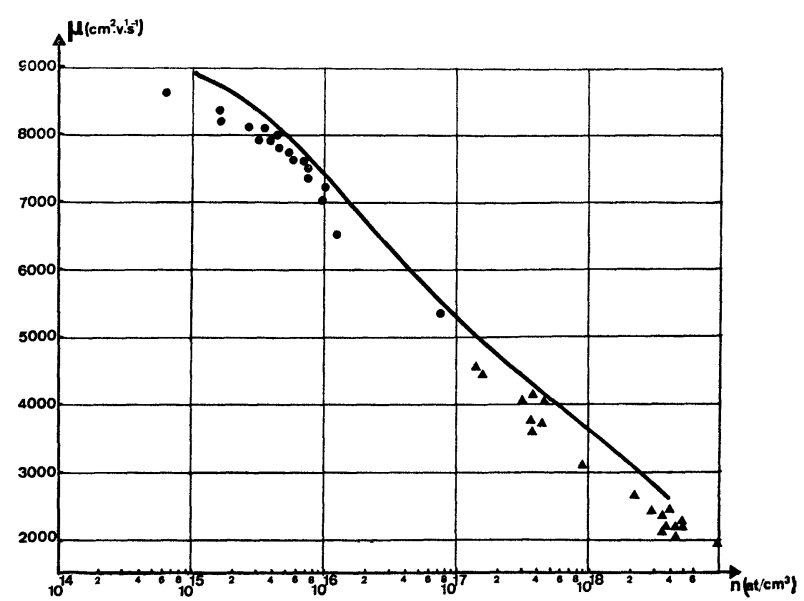

FIG. 8. - Caractéristiques mobilité-concentration des couches épitaxiales $\left(300^{\circ} \mathrm{K}\right)$. La courbe en traits pleins est la courbe théorique d'Ehrenreich [9]. 
Plusieurs remarques s'imposent :

a) Les caractéristiques des matériaux à profil de dopage plat et donnant de bons résultats en hyperfréquence se placent sur une courbe qui a bien l'allure de la courbe théorique, avec un léger décalage vertical par rapport à celle-ci.

b) Si les caractéristiques du matériau sont nettement au-dessous de cette courbe, plusieurs phénomènes peuvent en être la cause :

- ou bien les mesures d'effet Hall sont faussées par l'existence d'un profil de concentration important à l'intérieur de la couche,

- ou bien, si le profil est plat, le matériau est fortement compensé ou de mauvaise qualité cristalline.

Le taux de compensation $\eta=N_{D} / N_{A}$ peut être estimé à partir de mesures d'effet Hall à $77^{\circ} \mathrm{K}$ [7]. La figure 9 montre que la plupart de nos couches épitaxiales ont un taux de compensation compris entre 2 et 3,5 .

Les valeurs de $\eta$ donnent une idée globale de la compensation, mais il est possible d'étudier séparé-

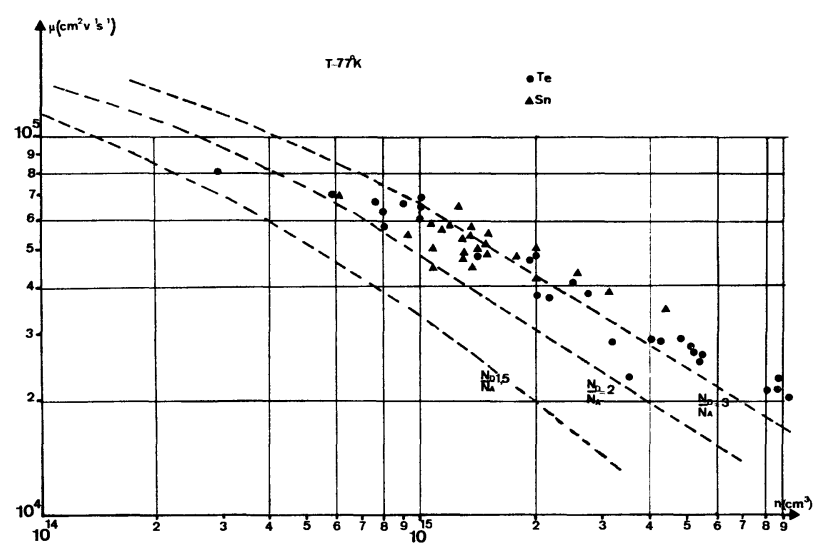

FIG. 9. - Caractéristiques mobilité-concentration des couches épitaxiales $\left(77^{\circ} \mathrm{K}\right)$. Les courbes en traits pointillés donnent le rapport de compensation $N D / N A$, d'après [7].

ment chaque centre de compensation par des méthodes photocapacitives [8]. Dans des couches très peu compensées, et à très faible concentration, on trouve seulement la présence de deux centres accepteurs : un niveau à $1,48 \mathrm{eV}$ au-dessous de la bande de conduction, attribué à un accepteur léger (Silicium), et un niveau à $1,2 \mathrm{eV}$ au-dessous de la bande de conduction, attribué à un complexe lacune de gallium-donneur.

Dans les couches plus compensées, on peut rencontrer en plus de ces deux centres, d'autres niveaux à $0,92 \mathrm{eV}, 0,85 \mathrm{eV}, 0,80 \mathrm{eV}, 0,75 \mathrm{eV}$, et le centre lié à l'oxygène à $0,67 \mathrm{eV}$.

A titre d'exemple, on trouvera sur les figures 10 et 11 , les courbes expérimentales des mesures photocapacitives effectuées sur les épitaxies Ep. II 307 et Ep. II 231 du tableau I. D'après les valeurs de mobilité

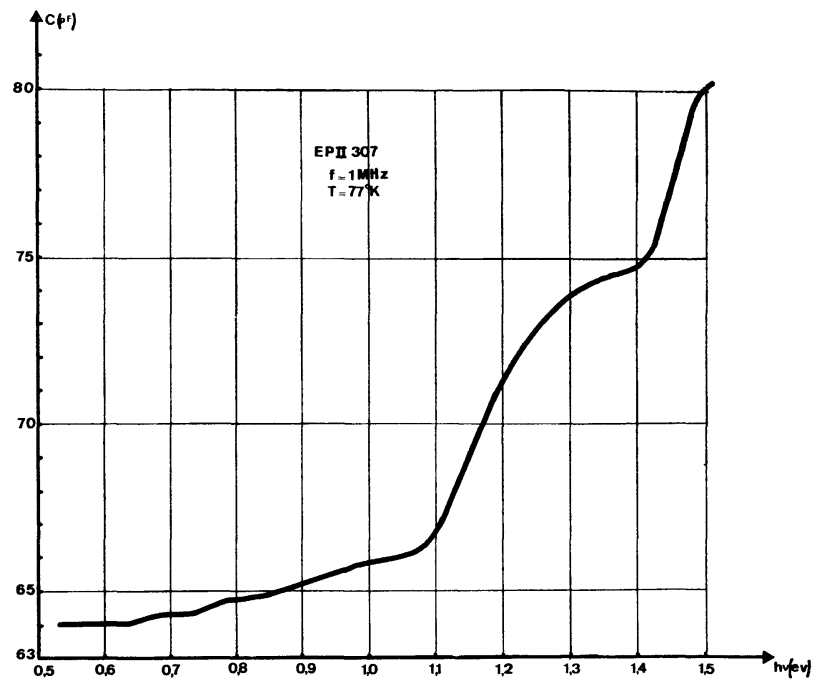

Fig. 10. - Mesures photocapacitives.

et de concentration mesurées à $300^{\circ} \mathrm{K}$ et à $77^{\circ} \mathrm{K}$, l'épitaxie Ep. II 321 est moins compensée que Ep. II 307. Le calcul donne :

$$
\begin{aligned}
& \eta=2,7 \text { pour l'Ép. II } 307 \\
& \eta=3,2 \text { pour l'Ép. II } 321
\end{aligned}
$$

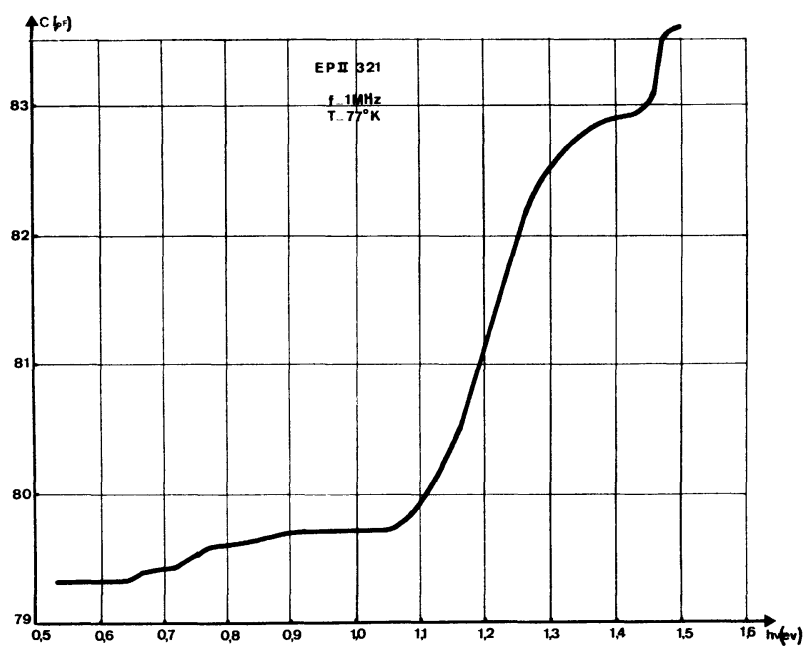

FIG. 11. - Mesures photocapacitives.

On remarque sur les courbes de mesures photocapacitives, que la différence de compensation entre ces deux matériaux est due surtout à l'apparition du centre à $0,92 \mathrm{eV}$ et à l'augmentation du peuplement des centres à $1,48 \mathrm{eV}$ et $1,20 \mathrm{eV}$, dans l'épitaxie Ep. II 307.

Conclusions. - La mise au point d'une méthode de dopage par rétrodiffusion permet de contrôler le niveau de dopage au cours de la croissance, et éventuellement, d'effectuer de brusques variations pour obtenir des structures multicouches.

L'interaction rapide des méthodes de caractérisation électrique avec les procédés d'élaboration des 
couches épitaxiales a permis d'obtenir des résultats importants sur le plan de la reproductivité et de l'homogénéité du dopage.
Les auteurs tiennent à exprimer leurs plus vifs remerciements à MM. Boulou et Grenet pour leur fructueuse collaboration.

\section{Bibliographie}

[1] Knight, Effer, Evans, Sol. St. Elec., 1965, 8, 178.

[2] ANdré (C.), Le Duc (J. M.), Boucher (A.), Hollan (L.), Journ. Intern. Micr. El. Toulouse, mars 1969.

[3] Boucher (A.), Hollan (L.), L'onde Elec., 1970, 50, 1.

[4] Boucher (A.), Brevet no 7.007.118.

[5] Schiller (C.), Hallais (J. P.) (à paraître).
[6] GREnet (R.), Brevet, demande de brevet en cours.

[7] Diguet (D.), Carballes (J. C.), Lebailly (J.), Conférence Dallas GaAs, 1968, 28.

[8] Fabre (E.), C. R. Acad. Sci., Paris, 1970, T270, 848.

[9] Ehrenreich (H.), Phys. Rev., 1960, 120, 1951. 